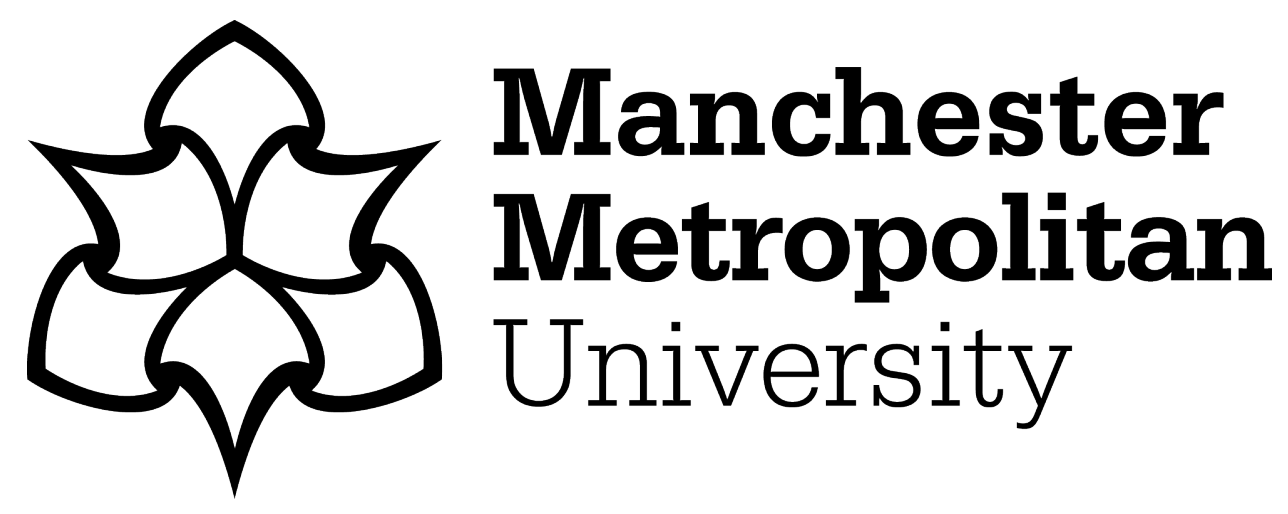

Till, Kevin, Morris, Rhys, Stokes, Keith, Trewartha, Grant, Twist, Craig, Dobbin, Nick, Hunwicks, Richard and Jones, Ben (2018) Validity of an Isometric Midthigh Pull Dynamometer in Male Youth Athletes. Journal of Strength and Conditioning Research, 32 (2). pp. 490-493. ISSN 1064-8011

Downloaded from: https://e-space.mmu.ac.uk/621559/ Version: Accepted Version

Publisher: National Strength \& Conditioning Association DOI: https://doi.org/10.1519/JSC.0000000000002324

Please cite the published version 


\title{
Validity of an Isometric Midthigh Pull Dynamometer in Male Youth Athletes
}

\author{
Kevin Till, ${ }^{1}$ Rhys Morris, ${ }^{1}$ Keith Stokes, ${ }^{2}$ Grant Trewartha, ${ }^{2}$ Craig Twist, ${ }^{3}$ Nick Dobbin, ${ }^{3,4}$ \\ RichaRd HuNwicks, ${ }^{4}$ AND Ben JONES ${ }^{1,4}$ \\ ${ }^{1}$ Institute for Sport, Physical Activity and Leisure, Leeds Beckett University, Leeds, United Kingdom; ${ }^{2}$ Department of Health, \\ University of Bath, Bath, United Kingdom; ${ }^{3}$ Department of Sport and Exercise Sciences, University of Chester, Chester, United \\ Kingdom; and ${ }^{4}$ Rugby Football League, Leeds, United Kingdom.
}

\section{Abstract}

Till, K, Morris, R, Stokes, K, Trewartha, G, Twist, C, Dobbin, N, Hunwicks, R, and Jones, B. Validity of an isometric midthigh pull dynamometer in male youth athletes. $J$ Strength Cond Res 32(2): 490-493, 2018-The purpose of this study was to investigate the validity of an isometric midthigh pull dynamometer against a criterion measure (i.e., $1,000-\mathrm{Hz}$ force platform) for assessing muscle strength in male youth athletes. Twentytwo male adolescent (age $15.3 \pm 0.5$ years) rugby league players performed 4 isometric midthigh pull efforts (i.e., 2 on the dynamometer and 2 on the force platform) separated by 5 -minute rest in a randomized and counterbalanced order. Mean bias, typical error of estimate (TEE), and Pearson correlation coefficient for peak force (PF) and peak force minus body weight $\left(\mathrm{PF}_{\mathrm{BW}}\right)$ from the force platform were validated against peak force from the dynamometer (DynoPF). When compared with $\mathrm{PF}$ and $\mathrm{PF}_{\mathrm{BW}}$, mean bias (with $90 \%$ confidence limits) for DynoPF was very large $(-32.4[-34.2$ to -30.6$] \%)$ and moderate $(-10.0[-12.8$ to -7.2$] \%)$, respectively. The TEE was moderate for both $\mathrm{PF}\left(8.1\right.$ [6.3-11.2] \%) and $\mathrm{PF}_{\mathrm{BW}}(8.9$ [7.0-12.4]). Correlations between DynoPF and PF $(r \quad 0.90$ [0.79-0.95]) and $\mathrm{PF}_{\mathrm{BW}}(r 0.90$ [0.80-0.95]) were nearly perfect. The isometric midthigh pull assessed using a dynamometer underestimated $\mathrm{PF}$ and $\mathrm{PF}_{\mathrm{BW}}$ obtained using a criterion force platform. However, strong correlations between the dynamometer and force platform suggest that a dynamometer provides an appropriate alternative to assess isometric midthigh pull strength when a force platform is not available. Therefore, practitioners can use an isometric midthigh pull dynamometer to assess strength in the field with youth athletes but should be aware that it underestimates peak force.

KEY WoRDS strength, peak force, adolescent, force platform

Address correspondence to Dr. Kevin Till, k.till@leedsbeckett.ac.uk 32(2)/490-493

Journal of Strength and Conditioning Research

(C) 2017 National Strength and Conditioning Association

\section{INTRODUCTION}

$\mathrm{M}$ uscle strength is an important physical quality for most sports (18) and has recently been advocated as a key training focus for young athletes (16). To compliment strength programming, the accurate assessment of muscle strength should be an important consideration for the practitioner working with young athletes. Maximal strength assessment methods typically include dynamic isoinertial measures using free-weight apparatus (e.g., one repetition maximum squat; (21) or resistance training machines (e.g., leg press; (19)). However, free-weight exercises require close supervision and should only be used when correct technical competency is demonstrated, limiting its use within younger and inexperienced groups, whereas resistance training machines lack applicability to sporting movements. To address these limitations, research has promoted the use of the isometric midthigh pull (IMTP) as an assessment of full body maximal strength in adults $(6,17,20)$ and youths $(3,8)$.

Isometric strength tests such as the IMTP involve an athlete applying maximal force to an immovable object. Strength qualities are quantified through the ground reaction forces using a force platform to attain measures such as peak force and rate of force development (18). Although numerous techniques have been used (e.g., knee angle and hip angle, $(2,20))$, studies have shown the IMTP to be a reliable strength-assessment method $(4,6,13)$. Relationships between IMTP strength and dynamic actions such as sprinting, (18), jumping (14), and weightlifting performance $(1,9)$ have been shown but are not consistent for all studies (15). Therefore, research to date recommends the IMTP as a useful method for assessing maximum strength. However, the utility of the method is likely to be limited by the availability of a force platform (18), which is often of high cost potentially limiting its use within youth athletes.

Recently, James et al. (13) compared the IMTP using a more cost-effective single axial loading cell against a force platform in recreationally active male adults. Results showed that the peak force obtained using the 2 devices was highly related; however, acceptable validity showed the single axial loading cell underreported peak force compared with the 
force platform. Therefore, the single axial loading cell offers an alternative IMTP assessment, but the validity and reliability within other populations (e.g., youth athletes) is unknown. Furthermore, although the single axial loading cell offers a force-time examination at a reduced cost, this still may not be accessible for all practitioners working with youth athletes. Instead, the development of a custom-built IMTP dynamometer offers a more cost-effective method for the measurement of full body maximal strength (5). However, for practitioners, it is important to understand the validity of any new device against the criterion method (10). Therefore, the purpose of this study was to compare the peak force obtained during the IMTP performed on a custom-built dynamometer (i.e., practical measure) and force platform (i.e., criterion measure) within male youth athletes.

\section{Methods}

\section{Experimental Approach to the Problem}

The study was designed to assess the validity of an IMTP exercise performed on a dynamometer against a force platform. All subjects underwent a familiarization session performing 2 IMTP attempts on the dynamometer and force platform, respectively. One week later, all subjects completed 2 IMTP assessments on the dynamometer and force platform in a randomized and cross-over design with 5-minute rest between efforts. The peak force obtained from the dynamometer was compared with that of the force platform.

\section{Subjects}

Twenty-two male adolescent rugby league players $( \pm$ SD age $15.3 \pm 0.5$ [age range: $14.5-16.0$ ] years, stature $177.9 \pm$ $5.0 \mathrm{~cm}$, and body mass $77.0 \pm 13.3 \mathrm{~kg}$ ) participated in this study. Ethics approval was granted by the Leeds Beckett University's ethics board and written informed consent, and parental consent was acquired from all subjects.

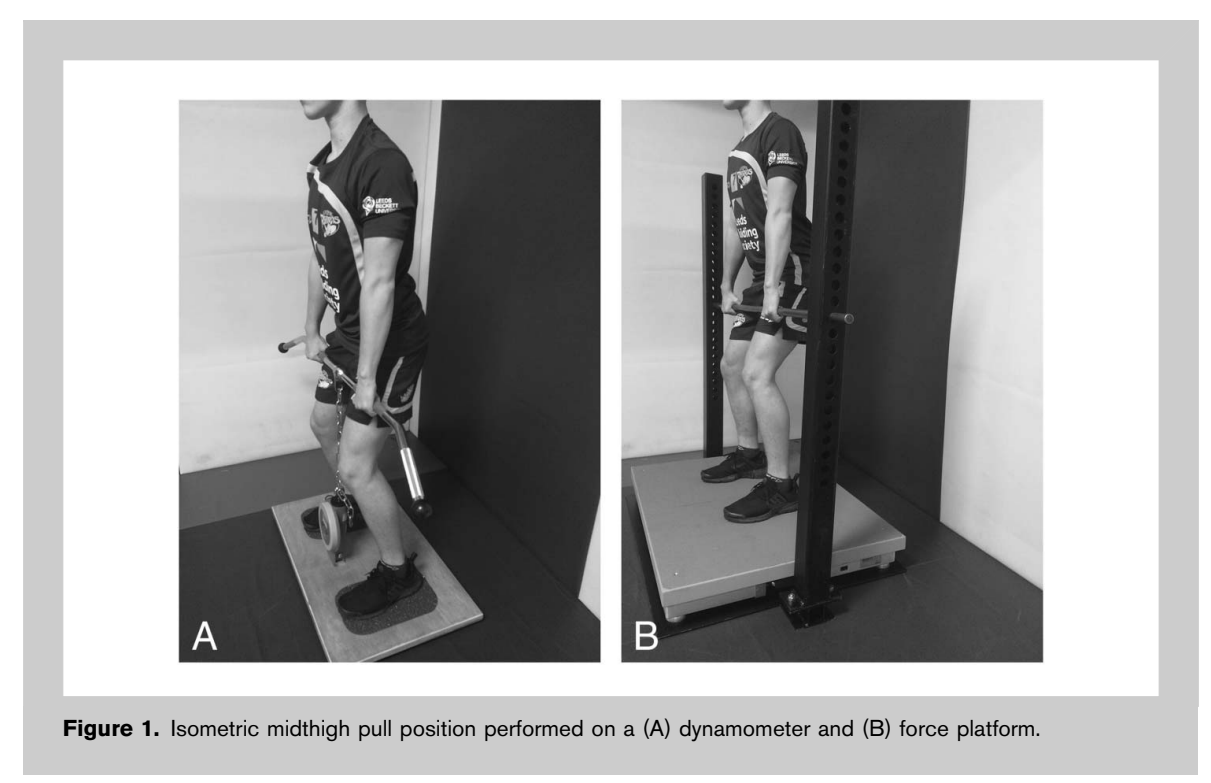

\section{Procedures}

The study was conducted during the preseason training phase with the testing session preceded by a standardized warm-up (light jogging, dynamic stretches, and submaximal isometric midthigh pull efforts). For the IMTP, subjects were positioned on each repetition to represent the second pull of the power clean with shoulders placed over the bar and feet hip width apart (Figure 1) consistent with previous studies $(7,13)$. Subjects were instructed to pull as hard and fast as possible after a 3 -second countdown as this is known to elicit the greatest peak force (1).

Dynamometer. A custom-built IMTP dynamometer was designed and built to include a T.K.K.5402 dynamometer (Takei Scientific Instruments Co., Ltd., Niigata, Japan), wooden platform $(80 \times 50 \mathrm{~cm})$ with rubber foot grips $(31 \times 20 \mathrm{~cm})$, chain $(51 \mathrm{~cm})$, and latissimus pulldown bar $(120 \mathrm{~cm}$; Decathlon, Stevenage, United Kingdom). The T.K. K.5402 dynamometer was removed from its original base $(31.5 \times 31.5 \mathrm{~cm})$ and attached to the wooden platform to allow subjects to adopt a wider foot position, with a wider bar allowing subjects to grip the bar representative of that during the second pull of the power clean. Subjects were positioned by standing on the foot grips and adjusting the chain length, so they were positioned in the above position. Subjects gripped the bar without the use of straps and prior to pulling maintained tension on the chain, so a jerk action was not performed. The dynamometer score was recorded in kilograms and then multiplied by 9.81 to represent a peak force dynamometer (DynoPF) value in Newton.

Force Platform. A commercially available portable force platform (AMTI, ACP, Watertown, MA, USA) with a sampling rate of $1,000 \mathrm{~Hz}$ was used as the criterion measure. The force plate base was $101 \times 80 \mathrm{~cm}$ with bar dimensions of $140 \times$ $3.3 \mathrm{~cm}$. Subjects were positioned as above by standing on the force platform and adjusting the bar height on a customized fixed rack, which enabled adjustments in bar height by 3 -cm increments, whereas further smaller adjustments were made by placing $1-\mathrm{cm}$ wooden boards on the force platform. The highest peak force $(\mathrm{PF})$ in Newton was used for analysis. In addition, peak force minus the subject's body weight $\left(\mathrm{PF}_{\mathrm{BW}}\right)$ was also used for analysis as the dynamometer did not measure the subject's body mass.

\section{Statistical Analyses}

All analysis was undertaken using an Excel spreadsheet (12). 
TABLE 1. Comparison of DynoPF, PF, and $\mathrm{PF}_{\mathrm{BW}}{ }^{*} \dagger$

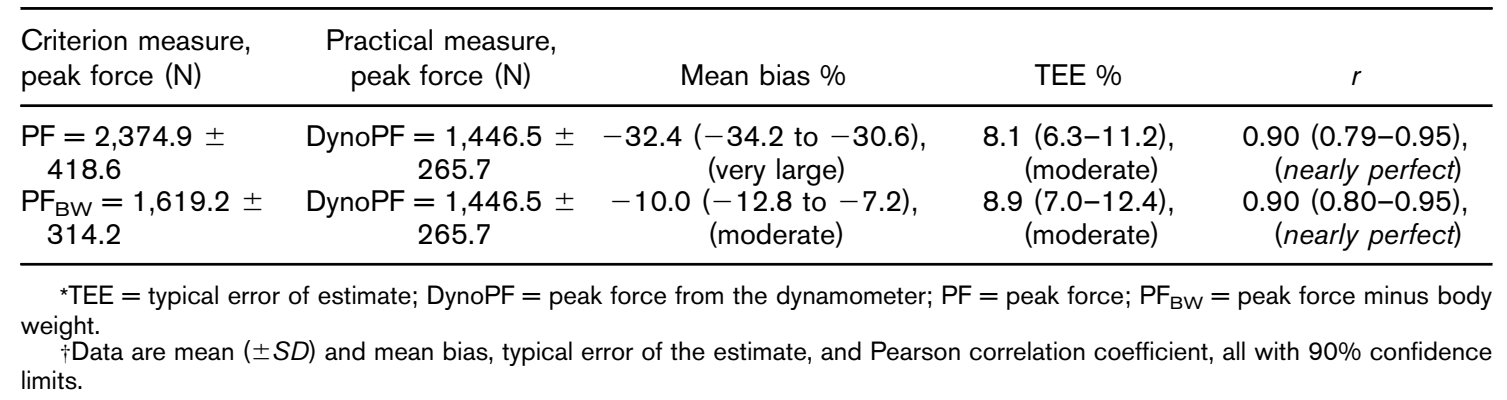

Within-session reliability was assessed using intraclass correlation coefficients (ICCs) and coefficient of variation $(\mathrm{CV})$. To analyze the validity between the dynamometer and force platform, mean bias, typical error of the estimate (TEE), and Pearson correlation coefficient were assessed, all with $90 \%$ confidence limits. Mean bias was rated as trivial $(<0.19)$, small $(0.2-0.59)$, medium $(0.6-1.19)$, or large (1.2-1.99). The TEE was rated as trivial $(<0.1)$, small $(0.1-0.29)$, moderate $(0.3-$ $0.59)$, or large $(>0.59)$. Correlations were rated as trivial $(<0.1)$, small $(0.1-0.29)$, moderate $(0.3-0.49)$, large $(0.5-$ $0.69)$, very large $(0.7-0.89)$, or nearly perfect $(0.9-0.99)(12)$. Linear regression analysis was used to determine a prediction equation to estimate the criterion measure from the practical measure along with regression statistics $\left(R^{2}\right)$. Using a $50 / 50$ split of the sample, a cross-validation of the prediction equations sought to establish whether there was minimal shrinkage in the $R^{2}$ value relative to the model.

\section{Results}

The intrasession reliability for the dynamometer was ICC $=$ 0.91 and $\mathrm{CV}=6.0 \%$. The intrasession reliability for the force platform was $\mathrm{ICC}=0.94$ and $\mathrm{CV}=4.3 \%$. The agreement between the IMTP PF and $\mathrm{PF}_{\mathrm{BW}}$ (criterion measures) with the DynoPF (practical measure) are shown in Table 1.

The regression analysis based on the cross-validation sample revealed that DynoPF explained 76.3\% (adjusted $R^{2}=0.76$ ) of the variance in $\mathrm{PF}$, yielding the equation $\mathrm{PF}=$ $(1.261 \times$ DynoPF $)+560.7$. Cross-validation analysis revealed a nonsignificant bias between the predicted and observed $\mathrm{PF}$, with an adjusted $R^{2}(78.2 \%)$. The overall regression model revealed that DynoPF explained $81.1 \%$ of the variance in $\mathrm{PF}$. The equation was $\mathrm{PF}=(1.300 \times$ DynoPF) +448.7 .

The regression analysis based on the cross-validation sample revealed that DynoPF explained 77.5\% (adjusted $\left.R^{2}=0.78\right)$ of the variance in $\mathrm{PF}_{\mathrm{BW}}$, yielding the equation $\mathrm{PF}_{\mathrm{BW}}=(1.091 \times$ DynoPF $)-9.15$. Cross-validation analysis revealed a nonsignificant bias between the predicted and observed $\mathrm{PF}_{\mathrm{BW}}$ with an adjusted $R^{2}$ (79.4\%). The overall regression model revealed that DynoPF explained $77.5 \%$ of the variance in $\mathrm{PF}$. The equation was $\mathrm{PF}_{\mathrm{BW}}=(0.972 \times$ DynoPF) +249.9 .

\section{Discussion}

This study compared the peak force obtained during the IMTP performed on a dynamometer (i.e., practical measure) with a force platform (i.e., criterion measure) in male youth athletes. Findings demonstrated that the IMTP dynamometer underestimated peak force on a force platform, when body weight was and was not included, identifying the dynamometer was not valid for assessing peak force. However, the good relative agreement between the dynamometer and force platform, as indicated by the near-perfect correlations, suggests that the dynamometer may be used as an alternative to a force platform for assessing IMTP with the regression equations applied to estimate PF.

Large differences between DynoPF and PF were apparent as a force platform measures the weight of an individual compared with the dynamometer measuring the "pull" force, as opposed to the ground reaction forces (18). Although this seems obvious, it is an important consideration as most studies (3) report absolute peak force from a force platform without considering body weight. However, when body weight was removed $\left(\mathrm{PF}_{\mathrm{BW}}\right)$, the dynamometer still underestimated peak force, which may have occurred because of the open-chain design of the dynamometer, which could increase the likelihood of force being applied outside the vertical axis (13) alongside increased proprioception requirements within the open-chained dynamometer compared with the closed-chain force platform (13). Furthermore, the grip on the dynamometer could have resulted in reduced peak force as the bar could more easily spin when gripped by the subjects compared with the fixed bar of the force platform. A further limitation is that body positions (e.g., joint angles) were not measured in each assessment and is therefore a limitation of the study. 
Although peak force was underestimated, nearly perfect correlations were observed suggesting that the dynamometer is able to measure IMTP strength and can discriminate between stronger and weaker individuals. Therefore, the dynamometer can be used to measure IMTP strength in youth athletes with the regression equations applied to calculate peak force. If the purpose is to assess muscle strength of large groups of athletes, especially within large field-based testing studies (11), the dynamometer seems a useful assessment method, especially considering the appropriate reliability.

Although this study suggests that a dynamometer can measure IMTP strength, the advantages of using a force platform should still be acknowledged. Force platform analysis allows force-time characteristics (e.g., impulse and rate of force development) to be assessed across varying time points (6), compared with the dynamometer providing a single maximum peak force. Therefore, when working with small athlete groups and a force platform is available, this would allow a more detailed profile of athletic performance.

In conclusion, IMTP strength assessed using a dynamometer-underestimated peak force obtained using a force platform, even when body weight was removed. The dynamometer is therefore not a valid measure of IMTP strength. However, nearly perfect correlations between the devices suggest that the dynamometer can discriminate between stronger and weaker individuals and can be used to assess IMTP strength in male youth athletes with regression equations applied to estimate peak force.

\section{Practical Applications}

An IMTP performed on a dynamometer is not a valid measure of peak force obtained from a force platform. However, the near-perfect correlations between the methods suggest that an IMTP dynamometer could be considered as part of a testing protocol. Practitioners should understand that peak force obtained using a dynamometer underestimate scores compared with a force platform, although regression equations can be used to estimate peak force. The IMTP is a safe, accessible, and efficient method for assessing strength when working with a large number of youth athletes where expensive laboratory-based equipment is not available.

\section{REFERENCES}

1. Beckham, G, Mizuguchi, S, Carter, C, Sato, K, Ramsey, M, Lamont H, Hornsby, G, Haff, G, and Stone, MH. Relationships of isometric mid-thigh pull variables to weightlifting performance. $J$ Sports Med Phys Fitness 53: 573-581, 2013.

2. Beckham, G, Sato, K, Mizuguchi, S, Haff, GG, and Stone, MH. Effect of body position on force production during the isometric mid-thigh pull. J Strength Cond Res, 2017. doi: 10.1519/ JSC.0000000000001968.

3. Darrall-Jones, JD, Jones, B, and Till, K. Anthropometric and physical profiles of English academy rugby union players. $J$ Strength Cond Res 29: 2086-2096, 2015.
4. De Witt, JK, English, KL, Crowell, JB Kalogera, KL, Guilliams, ME, Nieschwitz, BE, Hanson, AM, and Ploutz-Snyder, LL. Isometric mid-thigh pull reliability and relationship to deadlift 1RM. J Strength Cond Res 2016. Epub ahead of print.

5. Dobbin, N, Hunwicks, R, Jones, B, Till, K, Highton, J, and Twist, C. Criterion and construct validity of an isometric mid-thigh pull dynamometer for assessing whole body strength in professional rugby league players. Int J Sports Physiol Perform, 2017. doi: 10.1123/ ijspp.2017-0166.

6. Dos'Santos, T, Thomas, C, Jones, PA, and Comfort, P. Assessing muscle strength asymmetry via a unilateral stance isometric midthigh pull. Int J Sports Physiol Perform 6: 1-24, 2016.

7. Dos'Santos, T, Jones, PA, Kelly, J, McMahon, JJ, Comfort, P, and Thomas, C. Effect of sampling frequency on isometric midthigh-pull kinetics. Int J Sports Physiol Perform 11: 255-260, 2016.

8. Dos'Santos, T, Thomas, C, Comfort, P, McMahon, JJ, Jones, PA, Oakley, NP, and Young, AL. Between-session reliability of isometric mid-thigh pull kinetics and maximal power clean performance in male youth soccer players. J Strength Cond Res, 2017. doi: 10.1519/ JSC.0000000000001830.

9. Haff, GG, Carlock, JM, Hartman, MJ, Kilgore, JL, Kawamori, N, Jackson, JR, Morris, RT, Sands, WA, and Stone, MH. Force-time curve characteristics of dynamic and isometric muscle actions of elite women olympic weightlifters. J Strength Cond Res 19: 741-748, 2005.

10. Haugen, T and Buchheit, M. Sprint running performance monitoring: Methodological and practical considerations. Sports Med 46: 641-656, 2015.

11. Hislop, MD, Stokes, KA, Williams, S, McKay, CD, England, M, Kemp, SPT, and Trewartha, G. The efficacy of a movement control exercise programme to reduce injuries in youth rugby: A cluster randomised controlled trial. BMJ Open Sport Exerc Med 2: e000043, 2016

12. Hopkins, WG. Spreadsheets for analysis of validity and reliability. Sportscience 19: 36-42, 2015.

13. James, LP, Roberts, LA, Haff, GG, Kelly, VG, and Beckman, EM. Validity and reliability of a portable isometric mid-thigh clean pull. $J$ Strength Cond Res 38: 1378-1386, 2017.

14. Kawamori, N, Rossi, SJ, Justice, BD, Haff, EE, Pistilli, EE, O’Bryant, HS, Stone, MH, and Haff, GG. Peak force and rate of force development during isometric and dynamic mid-thigh pull cleans performed at various intensities. J Strength Cond Res 20: 483-491, 2006.

15. Kraska, JM, Ramsey, MW, Haff, GG, Fethke, N, Sands, WA, Stone, $\mathrm{ME}$, and Stone, $\mathrm{MH}$. Relationship between strength characteristics and unweighted and weighted vertical jump height. Int J Sports Physiol Perform 4: 461-473, 2009

16. Lloyd, RS and Oliver, JL. The youth physical development model: A new approach to long-term athletic development. Strength Cond J 34: 61-72, 2012.

17. McGuigan, MR, Newton, MJ, Winchester, JB, and Nelson, AG. Relationship between isometric and dynamic strength in recreationally trained men. J Strength Cond Res 24: 2570-2573, 2010.

18. McMaster, DT, Gill, N, Cronin, J, and McGuigan, M. A brief review of strength and ballistic assessment methodologies in sport. Sports Med 44: 603-623, 2014

19. Meylan, CM, Cronin, JB, Oliver, JL, Hughes, MM, Jidovtseff, B, and Pinder, $\mathrm{S}$. The reliability of isoinertial force-velocity-power profiling and maximal strength assessment in youth. Sports Biomech 14: 68-80, 2015

20. Stone, MH, Sands, WA, Carlock, J, Callan, S, Dickie, D, Daigle, K, Cotton, J, Smith, SL, and Hartman, M. The importance of isometric maximum strength and peak rate of force development in sprint cycling. J Strength Cond Res 18: 878-884, 2004.

21. Till, K, Tester, E, Jones, B, Emmonds, S, Fahey, J, and Cooke, C. Anthropometric and physical characteristics of English academy rugby league players. J Strength Cond Res 28: 319-327, 2014. 